\title{
Chapter 85 \\ Report on the Global Environment \\ Competitiveness of Lithuania
}

Lithuania is located in Northeastern Europe. It is situated along the southeastern shore of the Baltic Sea. It covers 62,670 square kilometres and borders to the east of Sweden and Denmark. It borders Latvia to the north, Belarus to the east and south, Poland to the south, and Russia to the southwest. Lithuania's climate, which ranges between maritime and continental, is relatively mild. It had a population of 3.03 million and domestic production the gross (GDP) of USD 42.7 billion in 2011. Through the evaluation of global environment competitiveness, we can know that the environment competitiveness index of Lithuania ranks at 55 among 133 countries.

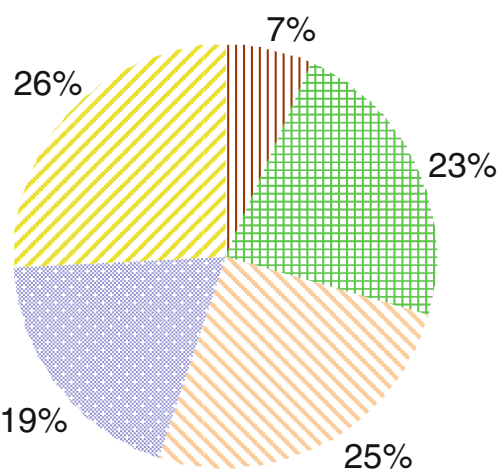

III Resource Environment Competitiveness

\# Ecological Environment Competitiveness Environment Carrying Competitiveness

* Environment Management Competitiveness

Fig. 85.1 Contribution of Environment Harmony sub-index of GEC Competitiveness 


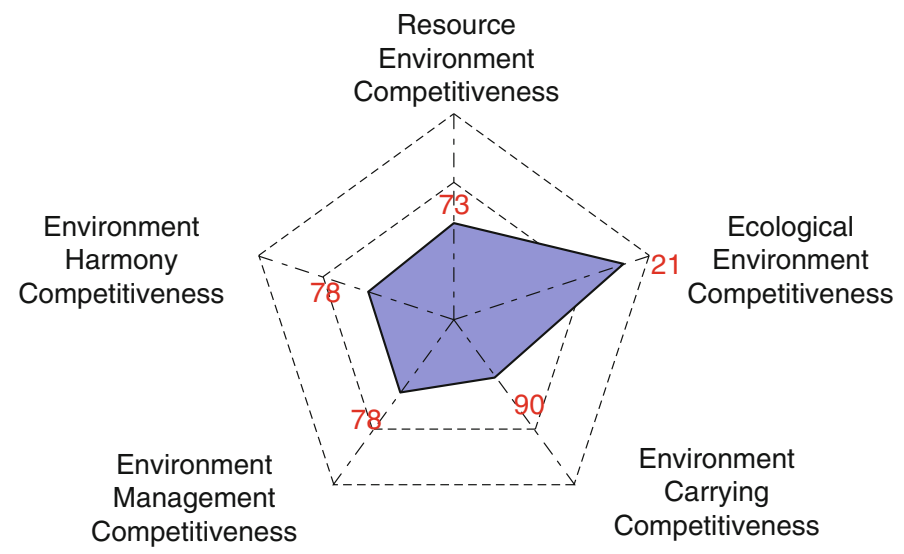

Fig. 85.2 Rank of sub-index of GEC

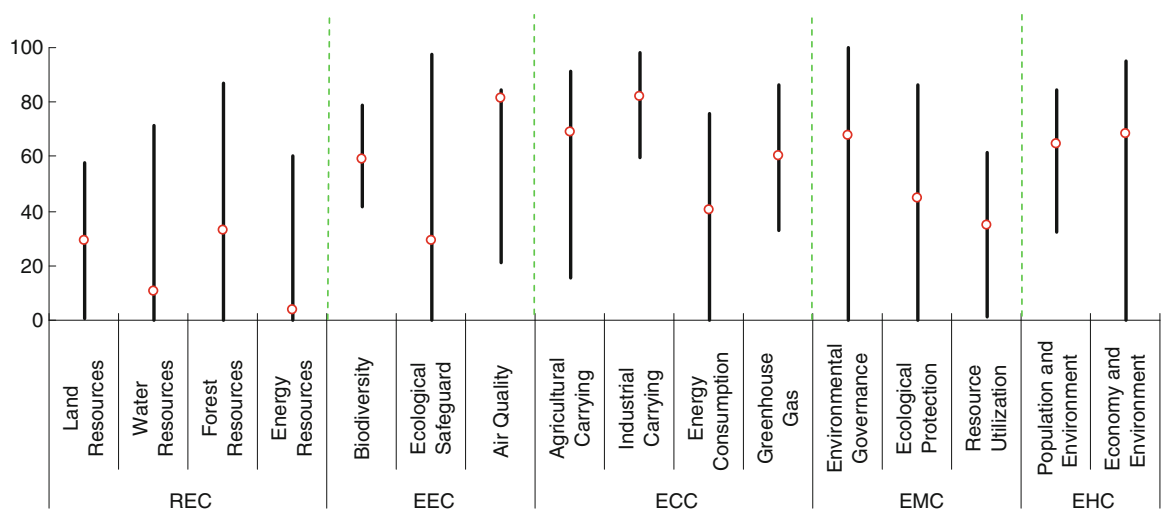

Fig. 85.3 Score and rank of the pillars of GEC

Table 85.1 Score and rank of all indicators of GEC

\begin{tabular}{|c|c|c|c|c|c|}
\hline Indicators & Score & Rank & Indicators & Score & Rank \\
\hline 1 Resource Environment & 16.79 & 73 & Groundwater & 2.72 & 97 \\
\hline Competitiveness & & & \multirow{2}{*}{$\begin{array}{l}\text { Total internal renewable } \\
\text { water resources }\end{array}$} & \multirow[t]{2}{*}{12.52} & \multirow[t]{2}{*}{71} \\
\hline 1.1 Land Resources & 29.35 & 12 & & & \\
\hline Land area per capita & 3.70 & 48 & 1.3 Forest Resources & 33.13 & 53 \\
\hline $\begin{array}{l}\text { Percentage of arable land } \\
\text { to total land area }\end{array}$ & 59.14 & 15 & $\begin{array}{l}\text { Growing stock in forest } \\
\text { and other wooded land }\end{array}$ & 51.42 & 62 \\
\hline Arable land per capita & 33.77 & 7 & \multirow{2}{*}{$\begin{array}{l}\text { Proportion of land area } \\
\text { covered by forest }\end{array}$} & \multirow[t]{2}{*}{40.51} & \multirow[t]{2}{*}{45} \\
\hline 1.2 Water Resources & 10.67 & 90 & & & \\
\hline Surface water & 4.25 & 63 & Forest area per capita & 4.99 & 32 \\
\hline Annual precipitation & 23.18 & 78 & 1.4 Energy Resources & 3.64 & 84 \\
\hline
\end{tabular}


Table 85.1 (continued)

\begin{tabular}{|c|c|c|c|c|c|}
\hline Indicators & Score & Rank & Indicators & Score & Rank \\
\hline Fossil energy & 0.00 & 64 & Energy consumption per unit & 99.77 & 68 \\
\hline Energy production & 0.47 & 88 & of land area & & \\
\hline $\begin{array}{l}\text { Proportion of combustible } \\
\text { renewables and waste to }\end{array}$ & 15.37 & 51 & $\begin{array}{l}\text { Ratio of clean energy } \\
\text { consumption }\end{array}$ & 59.60 & 6 \\
\hline $\begin{array}{l}\text { total energy consumption } \\
\text { Net energy imports of the }\end{array}$ & 2.66 & 111 & $\begin{array}{l}\text { Elasticity of energy } \\
\text { consumption }\end{array}$ & 0.00 & 126 \\
\hline energy consumption & 2.00 & & Elasticity of electric power & 2.08 & 121 \\
\hline $\begin{array}{l}2 \text { Ecological Environment } \\
\text { Competitiveness }\end{array}$ & 59.07 & 21 & $\begin{array}{l}\text { consumption } \\
\text { 3.4 Greenhouse Gas }\end{array}$ & 60.17 & 77 \\
\hline $\begin{array}{l}2.1 \text { Biodiversity } \\
\text { Threatened fish species }\end{array}$ & $\begin{array}{l}59.11 \\
97.17\end{array}$ & $\begin{array}{l}31 \\
17\end{array}$ & $\begin{array}{l}\text { Growth rate of } \mathrm{CO}_{2} \\
\text { emissions }\end{array}$ & 44.05 & 94 \\
\hline $\begin{array}{l}\text { Threatened mammal species } \\
\text { Threatened plant species }\end{array}$ & $\begin{array}{r}98.37 \\
100.00\end{array}$ & $\begin{array}{r}11 \\
1\end{array}$ & $\begin{array}{l}\text { Growth rate of Methane } \\
\text { emissions }\end{array}$ & 64.01 & 27 \\
\hline $\begin{array}{l}\text { GEF benefits index for } \\
\text { biodiversity }\end{array}$ & 0.00 & 128 & $\begin{array}{l}\mathrm{CO}_{2} \text { emissions per unit } \\
\text { of land area }\end{array}$ & 99.76 & 73 \\
\hline $\begin{array}{l}\text { 2.2 Ecological Safeguard } \\
\text { Terrestrial protected areas }\end{array}$ & $\begin{array}{l}29.11 \\
39.13\end{array}$ & $\begin{array}{l}47 \\
50\end{array}$ & $\begin{array}{l}\mathrm{CO}_{2} \text { emissions per unit of } \\
\text { energy consumption }\end{array}$ & 48.98 & 51 \\
\hline Marine protected areas & $\begin{array}{l}14.08 \\
8151\end{array}$ & 23 & $\begin{array}{l}4 \text { Environment Management } \\
\text { Competitiveness }\end{array}$ & 48.61 & 78 \\
\hline $\begin{array}{l}\text { 2.3 Air Quality } \\
\text { Inhalable particles (PM10) }\end{array}$ & $\begin{array}{l}81.51 \\
88.32\end{array}$ & $\begin{array}{l}16 \\
23\end{array}$ & $\begin{array}{l}\text { 4.1 Environmental } \\
\text { Governance }\end{array}$ & 67.50 & 111 \\
\hline $\begin{array}{l}\text { Particulate matter (PM2.5) } \\
\text { Index of indoor air pollution }\end{array}$ & $\begin{array}{r}87.21 \\
100.00\end{array}$ & $\begin{array}{r}52 \\
1\end{array}$ & $\begin{array}{l}\text { Agricultural chemicals } \\
\text { regulation }\end{array}$ & 95.24 & 20 \\
\hline Nitrogen oxides emission & 68.49 & 40 & $\begin{array}{l}\text { reguiation } \\
\text { Percentage of the rural }\end{array}$ & 0.00 & 122 \\
\hline $\begin{array}{l}\text { Sulfur dioxide emission } \\
3 \text { Environment Carrying } \\
\text { Competitiveness }\end{array}$ & $\begin{array}{l}40.88 \\
65.53\end{array}$ & $\begin{array}{l}40 \\
90\end{array}$ & $\begin{array}{l}\text { population with access } \\
\text { to an improved water }\end{array}$ & 0.00 & 122 \\
\hline 3.1 Agricultural Carrying & 69.24 & 51 & Percentage of the urban & 9800 & 70 \\
\hline $\begin{array}{l}\text { Cereal yield per unit of } \\
\text { arable land }\end{array}$ & 25.90 & 74 & $\begin{array}{l}\text { population with access } \\
\text { to an improved water }\end{array}$ & 30.00 & 10 \\
\hline Fertilizer consumption per & 96.33 & 54 & source & & \\
\hline unit of arable land & & & 4.2 Ecological Protection & 44.76 & 25 \\
\hline $\begin{array}{l}\text { Annual freshwater } \\
\text { withdrawals for }\end{array}$ & 99.95 & 15 & $\begin{array}{l}\text { Area of plantation and } \\
\text { afforestation }\end{array}$ & 0.68 & 48 \\
\hline agriculture per unit & & & Biome protect & 85.80 & 33 \\
\hline $\begin{array}{l}\text { of arable land } \\
\text { 3.2 Industrial Carrying }\end{array}$ & 82.16 & 118 & $\begin{array}{l}\text { Overfishing of fishing } \\
\text { resources }\end{array}$ & 62.50 & 32 \\
\hline $\begin{array}{l}\text { Net exports as a percentage } \\
\text { of GDP }\end{array}$ & 58.50 & 119 & 4.3 Resource Utilization & 34.85 & 75 \\
\hline Electric power consumption & 92.04 & 64 & $\begin{array}{l}\text { Utilization rate of water } \\
\text { resources }\end{array}$ & 0.38 & 61 \\
\hline $\begin{array}{l}\text { per unit of value added } \\
\text { of industry }\end{array}$ & & & $\begin{array}{l}\text { Percentage of total internal } \\
\text { renewable water }\end{array}$ & 60.08 & 75 \\
\hline $\begin{array}{l}\mathrm{SO}_{2} \text { emissions per unit of } \\
\text { value added of industry }\end{array}$ & 99.89 & 47 & $\begin{array}{l}\text { resources to total water } \\
\text { resources }\end{array}$ & & \\
\hline $\begin{array}{l}\text { Annual freshwater } \\
\text { withdrawals for industry }\end{array}$ & 78.21 & 124 & $\begin{array}{l}\text { Percentage of agricultural } \\
\text { land to total land area }\end{array}$ & 50.72 & 67 \\
\hline $\begin{array}{l}\text { per value added of } \\
\text { industry }\end{array}$ & & & $\begin{array}{l}\text { Percentage of fossil fuel } \\
\text { energy consumption to }\end{array}$ & 28.22 & 58 \\
\hline 3.3 Energy Consumption & 40.36 & 24 & total energy consumption & & \\
\hline
\end{tabular}


Table 85.1 (continued)

\begin{tabular}{|c|c|c|c|c|c|}
\hline Indicators & Score & Rank & Indicators & Score & Rank \\
\hline $\begin{array}{l}5 \text { Environment Harmony } \\
\text { Competitiveness }\end{array}$ & 66.51 & 78 & $\begin{array}{l}\mathrm{CO}_{2} \text { emissions (metric tons } \\
\text { per capita) }\end{array}$ & 89.20 & 76 \\
\hline $\begin{array}{l}\text { 5.1 Population and } \\
\text { Environment }\end{array}$ & 64.75 & 100 & $\begin{array}{l}\text { Energy consumption } \\
\text { per capita }\end{array}$ & 84.47 & 77 \\
\hline \multirow{2}{*}{$\begin{array}{l}\text { Improved sanitation facilities } \\
\text { (\% of population with } \\
\text { access) }\end{array}$} & 86.00 & 70 & $\begin{array}{l}5.2 \text { Economy and } \\
\text { Environment }\end{array}$ & 68.27 & 43 \\
\hline & 31.73 & 116 & $\begin{array}{l}\text { Land resource utilization } \\
\text { efficiency }\end{array}$ & 0.20 & 47 \\
\hline $\begin{array}{l}\text { Motor vehicles (per 1,000 } \\
\text { people) }\end{array}$ & 6.21 & 48 & $\begin{array}{l}\text { Sulfur dioxide emissions } \\
\text { per unit of GDP }\end{array}$ & 96.50 & 46 \\
\hline $\begin{array}{l}\text { Renewable internal } \\
\text { freshwater resources } \\
\text { per capita }\end{array}$ & & & $\begin{array}{l}\text { Carbon dioxide emissions } \\
\text { per unit of GDP }\end{array}$ & 87.87 & 67 \\
\hline $\begin{array}{l}\mathrm{SO}_{2} \text { emissions (metric tons } \\
\text { per capita) }\end{array}$ & 94.81 & 74 & $\begin{array}{l}\text { Energy consumption per unit } \\
\text { of GDP }\end{array}$ & 88.49 & 49 \\
\hline
\end{tabular}

Table 85.2 Rank distribution of the individual indicators of GEC

\begin{tabular}{lllllll}
\hline & $\begin{array}{l}\text { Number } \\
\text { of the } \\
\text { individual }\end{array}$ & Rank & Rank & Rank & Rank & Rank \\
indicators & $1-10$ & $11-30$ & $31-60$ & $61-100$ & $101-133$ \\
\hline Sub-index & 14 & 0 & 2 & 4 & 8 & 0 \\
\hline $\begin{array}{l}\text { Resource Environment } \\
\quad \text { Competitiveness }\end{array}$ & 11 & 2 & 4 & 5 & 0 & 0 \\
$\begin{array}{c}\text { Ecological Environment } \\
\quad \text { Competitiveness }\end{array}$ & 15 & 1 & 2 & 3 & 6 & 3 \\
$\begin{array}{c}\text { Environment Carrying } \\
\quad \text { Competitiveness }\end{array}$ & 10 & 0 & 2 & 2 & 4 & 2 \\
$\begin{array}{c}\text { Environment Management } \\
\text { Competitiveness }\end{array}$ & 10 & 0 & 0 & 4 & 5 & 1 \\
$\begin{array}{c}\text { Environment Harmony } \\
\text { Competitiveness }\end{array}$ & 60 & 3 & 10 & 18 & 23 & 6 \\
\hline Total & & & & & & \\
\hline
\end{tabular}

Open Access This chapter is distributed under the terms of the Creative Commons Attribution Noncommercial License, which permits any noncommercial use, distribution, and reproduction in any medium, provided the original author(s) and source are credited. 